\title{
The line-in-object superiority effect in perception: It depends on where you fix your eyes and what is located at the point of fixation
}

\author{
BRUCE EARHARD \\ Dalhousie University, Halifax, Nova Scotia B3H 4JI, Canada
}

\begin{abstract}
Williams and Weisstein (1978) reported that briefly presented line elements could be more accurately discerned in three-dimensional, coherent contexts than when presented alone. A series of five studies investigated this effect. The first study demonstrated that the general assumption that three-dimensional and coherent contexts are necessary conditions for demonstrating a line-in-object superiority effect is in error, and a robust effect can be demonstrated with contexts lacking coherence and depth. The remaining studies focused on a potentially more important determinant of the line-in-object effect. Evidence is presented that whether a given context facilitates, impairs, or has no effect on line detection is determined by the location of the fixation point and the amount of line detail at the fixation point. Proposals outlining processes assumed to underlie this fixation-location effect are advanced.
\end{abstract}

Weisstein and Harris (1974) demonstrated an objectsuperiority effect; that is, line elements could be more accurately discerned when they were part of a unitary, three-dimensional form than when part of a flat, less unified figure. More recently, McClelland (1978) and Williams and Weisstein (1978) have presented evidence for a line-in-object superiority effect, that is, line elements can be discriminated more readily when they are part of a coherent, three-dimensional form than when presented alone in the visual field. These studies are important because they question the viability of "bottom-up" feature analyzer models that assume perceptual discrimination of primitive features such as line elements as a preliminary step in the course of pattern perception. It seems clear that the structural configuration of a form as a whole can serve to facilitate or impair detection of the individual features or elements of which the form is composed. At a minimum, these data suggest that any proposed model of pattern perception has to be complex enough to permit higher order configurational structures to influence the discrimination of lower order features.

While the potential implications of these findings are substantial, before any systematic application of the findings to pattern perception theory can be made, it is necessary to determine the generality of

This research was supported by grants from the National Science Engineering Research Council of Canada and was presented at the Eastern Psychological Association, Philadelphia, April 1979. I am grateful to R. Armitage for running subjects and to J. Barresi, R. Klein, P. Jusczyk, and M. Ozier for their encouragement and advice. Requests for reprints should be addressed to B. Earhard, Department of Psychology, Dalhousie University, Halifax, Nova Scotia B3H 4JI, Canada. the line-in-object superiority effect. More specifically, can the effect be observed with all types of form or must forms possess certain properties to be effective? The possibility that three-dimensionality, connectedness, and coherence are important has been considered by a number of authors (e.g., Weisstein \& Maguire, 1980; Williams \& Weisstein, 1978). This view has been strengthened, on the one hand, by the findings of Womersley (1977), indicating that altering the forms used by Weisstein and Harris (1974) so as to render them less three-dimensional eliminated the object-superiority effect, and weakened, on the other hand, by the findings of McClelland and Miller (1979), indicating that three-dimensionality may not be as important a factor as has been commonly assumed.

In the first set of studies to be reported, we sought to clarify this issue by determining whether threedimensionality, coherence, and connectedness were necessary prerequisites for the demonstration of the line-in-object superiority effect. During the course of the investigation, it became apparent that they were not necessary, but that the location of the fixation point, or more specifically, the line detail located at the point of fixation, was critical in determining whether a specific context would give rise to the line-in-object superiority effect.

\section{EXPERIMENT 1}

\section{Method}

Apparatus and Stimuli. A Gerbrands two-field tachistoscope was used. In Experiment. $1 \mathrm{~A}$, the target-line elements shown in Figure $1 \mathrm{c}$ were presented alone, or in the context shown in Figure 1a, and in Experiment 1B, line targets were presented alone, or in the context shown in Figure $1 \mathrm{~b}$. Both contexts are effectively 


\section{a}
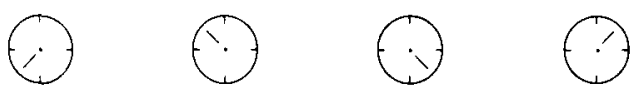

b
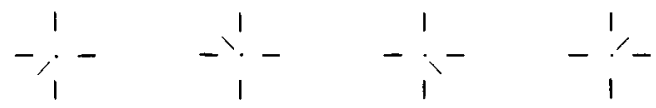

\section{C}

Figure 1. Target lines as they appeared in dial contexts (a), coordinate contexts (b), and alone (c) in Experiment 1.

flat, but that in Experiment $1 \mathrm{~A}$ (dial context) is a connected and coherent form, whereas that in Experiment $1 \mathrm{~B}$ (coordinate context) is unconnected and lacking in coherence, comprising only four shot line segments to serve as spatial reference points.

Stimuli were drawn with a Koh-i-nor Rapidomatic pen on white board. A .25-mm-diam nib was used to draw the target lines and the dial context, and a $.70-\mathrm{mm}$ nib was used to draw the reference lines to the coordinate context. The diameter of the dial context was $1.8 \mathrm{~cm}$. Reference lines used to make horizontal and vertical spatial direction in the coordinate context were $1.2 \mathrm{~cm}$ apart and $5 \mathrm{~mm}$ in length. The diagonal target lines were $5 \mathrm{~mm}$ in length and were removed $2 \mathrm{~mm}$ from the exact central point of the context so as to ensure that the fixation point did not fall directly upon the end of the target line elements to be detected. The viewing distance was $60 \mathrm{~cm}$, and the visual angles subtended by the dial context, the coordinate context, and the target line elements were $1.62,2.10$, and $.48 \mathrm{deg}$, respectively. A very faint pencil dot, approximately $.35 \mathrm{~mm}$ in diameter, served as a fixation point.

Procedure. Subjects fixated the pencil dot and, when instructed to proceed, pressed a button initiating the removal of the preexposure field and a $20-\mathrm{msec}$ presentation of the exposure field containing stimulus material (but no fixation point), followed by the immediate reappearance of the preexposure field. Prior to the start of the presentation sequence, the subjects were provided with examples of the stimulus materials to be exposed. They were informed that the object of interest was their ability to detect the target line elements; they indicated which of the four alternative target lines had been shown after each presentation by pointing at it on a display card. The level of illumination of the exposure field was fixed at $3.38 \mathrm{~cd} / \mathrm{m}^{2}$; that of the preexposure field was adjusted for every subject so as to ensure an overall performance level of $70 \%-80 \%$ correct responding (mean levels were 5.83 and $4.97 \mathrm{~cd} / \mathrm{m}^{2}$ for $A$ and $B$, respectively).

Two blocks of 32 practice presentations familiarized subjects with procedures and allowed adjustments to the appropriate level of illumination of the preexposure field. Twelve additional blocks of 32 test presentations followed. In each presentation block, on half the trials the line elements appeared alone and on the other half appeared in either the dial or coordinate context, depending upon the experiment. The order of items within a presentation block was random, with the restriction that no more than four presentations of any one type (context or line alone), or line orientation, could occur in immediate succession. The subjects were given no feedback about their performance level. Eight subjects were used in both Experiments $1 \mathrm{~A}$ and $1 \mathrm{~B}$. Two subjects served in both experiments. All were naive with respect to the objective of the study.

\section{Results}

The subjects were more accurate in specifying the target line when it was presented within either the dial or coordinate context. In Experiment 1A, target lines placed within a context were reported accurately on $81.3 \%$ of the presentations, whereas lines alone were reported accurately on $70.6 \%$ of the presentations. The corresponding figures for Experiment $1 \mathrm{~B}$ are $81.3 \%$ and $71.3 \%$, respectively. The advantage of the line in context was evident in the data of each subject, ranging from $4.7 \%$ to $23.4 \%$ in Experiment $1 \mathrm{~A}$ and from $4.7 \%$ to $21.9 \%$ in Experiment 1B. A $t$ test applied to the mean number of correct responses on target lines within a context vs. mean number correct on target lines alone showed significantly better performance for the context format in Experiment $1 \mathrm{~A}[\mathrm{t}(7)=5.21, \mathrm{p}<.01]$ and in Experiment $1 \mathrm{~B}[\mathrm{t}(7)=4.56, \mathrm{p}<.01]$. Moreover, the overall advantage of the context format of $10.7 \%$ in Experiment $1 \mathrm{~A}$ and $10.0 \%$ in Experiment $1 \mathrm{~B}$ is similar to the $11.2 \%$ advantage reported by Williams and Weisstein (1978) in their comparisons of diagonal target lines presented alone and within a context.

The experiments demonstrate that a substantial line-in-context effect can be produced with the forms employed. It remains to be shown that the forms are, indeed, lacking in three-dimensionality and coherence. The relevant information is provided in the Appendix, which shows depth and coherence ratings of these forms by independent groups of subjects. Depth and coherence can be most reasonably assessed by comparing the ratings of the forms used in our study with (1) the best, coherent, threedimensional object context and (2) the poorest, least effective, line context (assumed to be lacking in depth and coherence), typically used in studies of the object superiority and the line-in-context effect (see Williams \& Weisstein, 1978, Figures 1a and 1d, respectively). Reference to the Appendix will show that both the dial and the coordinate contexts were rated very much flatter than the three-dimensional object form $[\mathrm{t}(44)=20.95, \mathrm{p}<.001$, and $\mathrm{t}(44)=17.10$, $\mathrm{p}<.001$, respectively] and not different from or, in the case of the dial context, flatter than the line context $[\mathrm{t}(44)=.64, \mathrm{p}>.05$, and $\mathrm{t}(44)=3.63, \mathrm{p}<.05$, respectively]. The dial context was as coherent as the object context $[t(44)=1.75, p>.05]$, and both were more coherent than the line context $[t(44)=6.15$, $\mathrm{p}<.01$, and $\mathrm{t}(44)=7.85, \mathrm{p}<.01$, respectively]. The coordinate context was rated as no more coherent than the line form $[t(44)=.16, p>.05]$ and much less coherent than either the dial or object form $[\mathrm{t}(44)=7.10, \mathrm{p}<.001$, and $\mathrm{t}(44)=8.42, \mathrm{p}<.001$, respectively]. In short, the dial context was assigned a high coherence rating and the coordinate context a very low coherence rating. Both contexts received low depth ratings. Obviously, the presence or ab- 
sence of coherence does not appreciably alter the magnitude of the line-in-context effect, and the effect can be produced by contexts extremely deficient in three-dimensionality.

\section{EXPERIMENT 2}

Experiment 1 indicates that a pronounced line-incontext superiority effect can be produced with forms rated extremely low in depth and coherence. Experiment 2 was undertaken to strengthen the generality of that conclusion by demonstrating that the stimulus parameters and procedures of Experiment 1 were not unique and would be equally effective in producing a line-in-context effect with the threedimensional stimuli used by Williams and Weisstein (1978). A preliminary study used the Williams and Weisstein object form with the fixation point (Figure 2a) to make conditions as comparable as possible to Experiment 1. Rather surprisingly, the study did not show any advantage associated with presenting the line in context. To confirm the preliminary finding and to determine the possible importance of the fixation point, three experiments were carried out. In Experiment $2 \mathrm{~A}$, the fixation point was located in the

\section{SET A SET B}

a
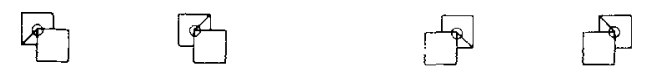

b
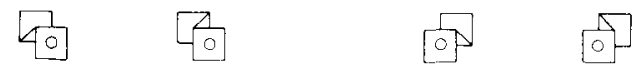

ro

C
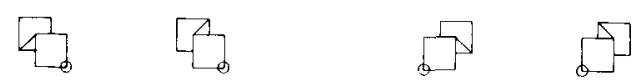

Figure 2. Contexts and target lines from Williams and Weisstein (1978) separated into Sets $A$ and $B$ for use in Experiment 2. Contexts and target lines with fixation point locations shown in Rows a, b, and c were used in Experiments 2A, 2B, and 2C, respectively. Circles are included only to clarify changes in position of fixation point, and were not part of the stimulus displays. See text for details on fixation point. same position as in the preliminary study, as shown in Figure 2a. In Experiment 2B, the fixation point was moved to the central area of the forward square of the form, exactly the same position used by Williams and Weisstein, as shown in Figure $2 \mathrm{~b}$. In Experiment $2 \mathrm{C}$, it was moved still further to the location shown in Figure 2c.

\section{Method}

Stimuli. We wished to retain the arrangement of target lines radiating from a central fixation point used in Experiment 1, but under such circumstances, if all four variants of the object form are used, the location of the form is confounded with the identity of the target line. This problem can be resolved by using only the two variants of the object form classed as Set A in Figure 2. The position of the fixation point and location of the context form remain fixed, and two of the four diagonal target lines contained in these contexts can be compared with the same lines presented alone. Shifting the location of the forward square to the left produces the context forms shown in Set B of Figure 2 with the diagonal target-line elements in the opposite orientation. By presenting half the subjects with context forms from Set A, and the other half with context forms from Set B, it was possible to present the four diagonal lines used in Experiment 1 both alone and within the object context. This procedure converts the fouralternative choice task used in Experiment 1 into a two-alternative, forced-choice task, but Williams and Weisstein have shown (their Experiment 2) that a two-alternative choice task incorporating these forms and line elements produces a large object-line superiority effect. Context forms and target line elements were drawn by using a Rapidomatic pen with a .25 - $\mathrm{mm}$ nib. The target line elements and context subtended visual angles of .56 and $1.14 \mathrm{deg}$, respectively.

Subjects and Procedure. Eight naive subjects were employed in Experiments $2 \mathrm{~A}$ and $2 \mathrm{C}$, and 10 in Experiment 2B. No subject served in more than one experiment, and none had participated in Experiment 1. In all experiments, half the subjects received context and line stimuli from Set $\mathbf{A}$ and half received them from Set B. As in preceding studies, line elements alone and in context were combined randomly, in equal numbers, into 32 -item presentation blocks. At least two blocks of practice presentations preceded 12 blocks of test presentations. During practice presentations, the level of illumination of the preexposure field was adjusted for each subject so as to ensure an overall performance level of $70 \%-80 \%$ correct responding (mean levels were 7.03, 5.83 , and $4.97 \mathrm{~cd} / \mathrm{m}^{2}$ in $\mathrm{A}, \mathrm{B}$, and $\mathrm{C}$, respectively). The level of illumination of the exposure field and the exposure duration was the same as in Experiment 1.

\section{Results}

In Experiment $2 \mathrm{~A}$, with the fixation point located at the junction of the target line and two other context lines (Figure 2a), it was found, as in the preliminary study, that the anticipated object-line superiority effect was not evident. In fact, the line alone was discerned more accurately. On the average, the line alone was reported accurately on $81.7 \%$ of the presentations and the line in context, on $69.5 \%$ of the presentations. The advantage associated with line alone was evident in the data of each subject, its magnitude ranging from $6.3 \%$ to $21.4 \%$, and was reliable $[\mathrm{t}(7)=5.80, \mathrm{p}<.001]$. This is contrary to results reported by Williams and Weisstein (1978) with such a form. The discrepancy could reflect procedural dif- 
ferences or differences in location of the fixation point. Experiment $2 \mathrm{~B}$ confirms that it is the latter factor that is crucial.

In Experiment 2B, the fixation point was in the same position as that used by Williams and Weisstein, and the results were as they report: Target lines were detected more accurately when presented within the object context than when presented alone. On the average, the subjects were correct on $81.8 \%$ of the presentations when the line elements were part of a context and on only $69.8 \%$ when they were presented alone. Nine of 10 subjects were more accurate when the line was contained within the context (range $5.2 \%-19.8 \%$ ). The line-in-context advantage is reliable $[\mathrm{t}(9)=6.03, \mathrm{p}<.001]$, and the size of the overall difference of $12.0 \%$ is similar in magnitude to that reported by Williams and Weisstein. It is evident that, with our procedure and stimuli, the results of Williams and Weisstein can be replicated as long as the fixation point is appropriately located.

In Experiment $2 \mathrm{C}$, moving the fixation point still farther away from the initial position, to the location shown in Figure $2 c$, eliminated the context advantage evident in Experiment 2B. The overall difference of $4.2 \%$ in favor of the line alone was small and not reliable $[t(7)=1.2, p>.05]$.

\section{Discussion}

Experiment 2 shows that moving the fixation point altered context effectiveness dramatically. Why should this be so? It is not because the movement of the fixation point produced changes in apparent depth of the context. The Appendix shows that when subjects were asked to assign depth ratings with the fixation point positioned at the different locations used in Experiment 2, their overall rating of the object form was slightly lower than when a fixation point was not used, but there was little systematic change in apparent depth with changes in fixation point location. Differences were small and not reliable $[\mathrm{F}(2,88)=1.89, \mathrm{p}>.05, \mathrm{MSe}=40.61]$.

Another possible explanation is that masking transients produced by fixation point offset interact with additional line elements present at the fixation point to impair performance in Experiments $2 \mathrm{~A}$ and $2 \mathrm{C}$. However, when Experiment $2 \mathrm{~A}$ was repeated, with a single change in procedure-namely, the fixation point was always present-the same advantage of line-alone over line-in-context was found. Its magnitude was $10.4 \%[t(7)=4.83, p<.001]$ compared with $12.2 \%$ in Experiment $2 \mathrm{~A}$.

It does seem likely, however, that additional line elements present at the point of fixation in Experiments $2 \mathrm{~A}$ and $2 \mathrm{C}$ do in some way impair context effectiveness. Certainly, the main respect in which these experiments differ from Experiment $2 \mathrm{~B}$ is that, in the latter, the fixation point falls on a central area free of line detail. The amount of line detail is espe- cially pronounced in Experiment $2 \mathrm{~A}$, where the fixation point is located at the junction of target and context lines, and it is in this experiment that it is most disadvantageous to have the target line in the context. If it is the concentration of line elements at the point of fixation, and about the target line, which produces the difficulty in detecting the target line, then it should be possible to alter forms used as contexts so as to make target line detection either easier, or more difficult, than would be the case if target lines were presented alone. In Experiment 1, for example, the fixation point was located close to the point where target lines terminated, but context lines were well removed from the point of fixation, and context facilitation of target line detection was evident. If the target and context lines were moved inward toward the point of fixation, would target line detection be impaired? Experiment 3 was designed to answer this question.

\section{EXPERIMENT 3}

Experiment 3 used coordinate context forms of the type used in Experiment 1B, shown in Figures 3a and $3 \mathrm{~b}$. The large context shown in Figure $3 \mathrm{a}$ is most similar to the form used in Experiment 1B. The target line is isolated from the coordinate reference lines and the fixation point, so that the context facilitation of target line detection in Experiment 1 should also be evident here. In the small context shown in Figure $3 \mathrm{~b}$, the reference lines are much closer to the point of fixation (and the target line). We expected that this proximity would neutralize the effect of reference context and perhaps permit target line elements to be discerned more accurately when presented alone.

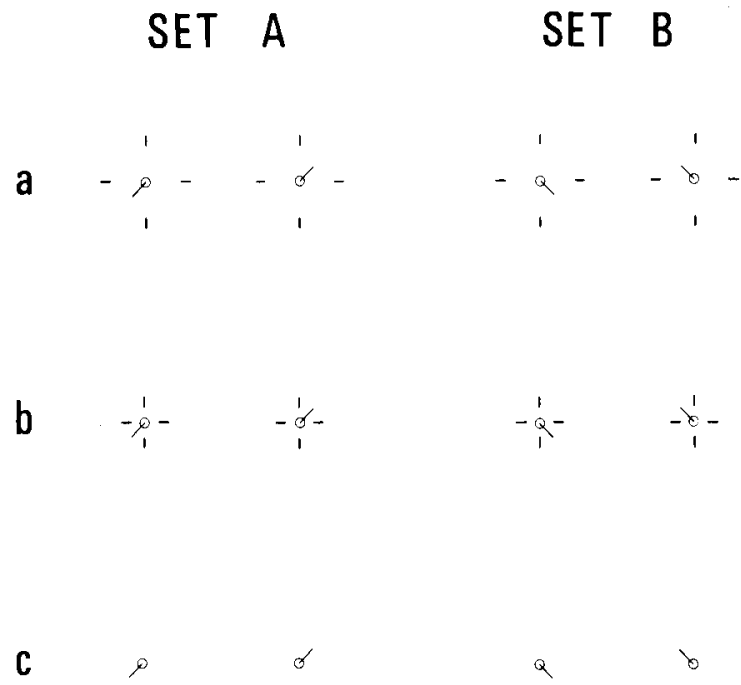

Figure 3. Target lines from Sets $A$ and $B$ as they appeared in large coordinate contexts (a), small coordinate contexts (b), and alone (c) in Experiment 3. Circles are included only to clarify location of fixation point, and were not part of stimulus displays. 


\begin{abstract}
Method
Stimuli. A .50-mm nib was used to draw the coordinate context. Reference lines were $3 \mathrm{~mm}$ in length and were separated by $2 \mathrm{~cm}$ and $8 \mathrm{~mm}$ in large and small contexts, respectively. The visual angles subtended by large and small contexts were 2.48 and $1.34 \mathrm{deg}$, respectively. Target lines were drawn with a $.25-\mathrm{mm}$ nib and, as in Experiment 2, were anchored exactly at the point of fixation and subtended a visual angle of $.56 \mathrm{deg}$.

Subjects and Procedure. Only four naive subjects were presented with the large coordinate figure and target line elements because this part of the experiment was viewed as a partial replication of Experiment 1. Eight additional naive subjects were presented with the small coordinate context and target line elements. No subject had served in any of the preceding experiments. Half of the subjects were presented with context and line elements from Set A in Figure 3, and half with those from Set B. Within each of 12 presentation blocks, target line elements from the appropriate set appeared alone and in context equally often. The amount of practice, stimulus presentation procedures, exposure duration, illumination level of the exposure field, and procedures used to alter illumination level of the preexposure field to maintain a performance level of $70 \%-80 \%$ correct responding were as described in preceding studies. The mean levels of illumination of the preexposure field were 6.72 and $7.72 \mathrm{~cd} / \mathrm{m}^{2}$ for large and small coordinate contexts, respectively.
\end{abstract}

\section{Results}

The data obtained with the large coordinate context were similar to those obtained in Experiment 1. On the average, subjects detected the presence of a target line in context on $84.0 \%$ of the presentations; they reported them as being alone on only $67.4 \%$ of the presentations. All four subjects showed the advantage of context (with differences ranging from $10.4 \%$ to $29.6 \%$ ). The overall mean difference of $16.6 \%$ favoring context was reliable $[\mathrm{t}(3)=3.72, \mathrm{p}<.05]$ and somewhat larger than that observed in Experiment 1.

The data obtained with the small coordinate context were quite different. Line targets were detected more accurately alone $(79.1 \%)$ than when contained within the context $(72.2 \%)$. The advantage of line alone over line in context ranged from $2.1 \%$ to $12.0 \%$ for individual subjects. The overall advantage of $6.9 \%$ favoring the line alone over the line in context was small, but reliable $[\mathrm{t}(7)=5.89, \mathrm{p}<.001]$.

Experiment 3 shows that if the reference lines in the large coordinate context that facilitates target line detection are caused to converge upon the point of fixation (and the target line), the advantage associated with the context is lost and, just as in the case of the William and Weisstein form, target lines can be detected more accurately alone. Observed differences cannot be attributed to variations in depth or coherence of the forms. Reference to the Appendix shows that the small form, which was the inferior context, was rated higher than the large context in both depth $[\mathrm{t}(44)=2.58, \mathrm{p}<.05]$ and coherence $[\mathrm{t}(44)=2.54, \mathrm{p}<.05]$. Overall, both the small and large contexts were rated extremely low on both scales and did not differ from the Weisstein and Williams line context in either depth $[t(44)=1.63$, $\mathrm{p}>.05$, and $\mathrm{t}(44)=.34, \mathrm{p}>.05$, respectively] or coherence $[\mathrm{t}(44)=.08, \mathrm{p}>.05$, and $\mathrm{t}(44)=1.28$, p > .05, respectively].

Experiment 3 supports our conjecture that the convergence of additional line elements on the target line and the point of fixation is the major factor contributing to the difficulty in discerning target lines in the Williams and Weisstein form shown in Figure 2a. Experiment 3 does not, however, address a troublesome other question raised by the results of Experiment 2 . Why does locating the fixation point at the junction of intersecting line elements, as shown in Figure 2a, impair line detection, whereas positioning the line at the center of the open forward square of the form, as in Figure 2b, facilitates detection of target line elements? If it is the presence of additional lines converging upon and joining the target line that impairs target line detection, why should the effect vary with fixation point location? Moving the fixation point $.54 \mathrm{deg}$ to the central area of the forward square in the Williams and Weisstein form in Figure 2 does not alter the fact that the additional lines continue to converge upon the target line, and yet, after such a movement of the fixation point, there is facilitation rather than impairment of target line detection. It seems that interference or impairment produced by the proximity of additional line elements to target lines in Experiment 2 is dependent upon additional line elements and target lines being anchored at, or converging upon, the point of fixation. Experiments 4 and 5 sought to clarify this issue.

\section{EXPERIMENT 4}

Experiment 4 sought further evidence that the facilitation of target detection by context can be eliminated or reversed by a small shift in fixation point. The context, extracted from the Williams and Weisstein object form, consisted of the two line segments that joined with the target line and that were assumed to contribute to the difficulty in discerning the target line when fixation was at the junction of target and context lines in Experiment $3 \mathrm{~A}$. Depth ratings are provided in the Appendix. The form was rated intermediate in depth, lower than the three-dimensional object form $[\mathrm{t}(44)=9.98$, $\mathrm{p}<.001]$, but higher than the flat line context [t(44) $=7.37, \mathrm{p}<.001] .{ }^{1}$ It received low coherence ratings and did not differ from the line context $[\mathrm{t}(44)=.19$, $p>.05]$. Figure 4a shows the fixation point located at the junction of target and context lines. Figure $4 b$ shows the fixation point moved the same distance away from the point of intersection and target lines as that used by Williams and Weisstein when presenting the full object form. The issue of interest was whether context would facilitate target detection in the latter arrangement but not in the former. 
SET A

SET B

a
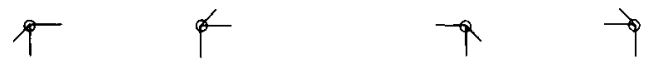

$$
\rho
$$$$
\sigma
$$$$
Q
$$$$
b
$$

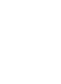

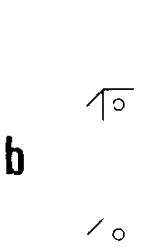

Figure 4. Target lines from Sets $A$ and $B$ are shown as part of the corner context used in Experiment 4 with the fixation point at the junction of target and context line units (a) and removed to the location normally used with the full Williams and Weisstein form (b). Immediately below each context, target lines presented alone, appropriately positioned with respect to fixation point, are shown. Circles are included only to clarify fixation point location; they were not part of the stimulus display.

\section{Method}

Stimulus materials. Contexts and target line elements were drawn with a , 25-mm nib. They were the same length as in Experiment 2, in which the full Williams and Weisstein form was used, and subtended visual angles of .76 and $.56 \mathrm{deg}$, respectively.

Subjects and Procedures. Eight naive subjects were presented with target lines alone and in context, with the fixation point location shown in Figure $4 a$, and eight additional naive subjects, the same materials with the fixation point location shown in Figure 4b. None of the subjects had served in any of the preceding experiments. Half the subjects were presented with lines and contexts from Set $A$, and the other half, from Set B in Figure 4. Target lines appeared alone and in context equally of ten in random order in each of the 1232 -item presentation blocks. The amount of practice and stimulus presentation procedures were as in preceding studies. The mean level of illumination of the preexposure field to maintain the $70 \%-80 \%$ level of correct responding was 5.83 and $4.72 \mathrm{~cd} / \mathrm{m}^{2}$ for the fixation point locations shown in Figures $4 \mathrm{a}$ and $4 \mathrm{~b}$, respectively.

\section{Results}

When the fixation point was located away from the point at which the target line and context line joined, as in Figure 4b, the subjects detected line elements within the context much more accurately than line elements presented alone. On the average, the subjects detected target lines correctly when they were part of the context on $86.0 \%$ of the presentations, but on only $66.4 \%$ when they were presented alone. Seven of the eight subjects favored the line in context over line alone (range 8.3\%-30.2\%). The mean difference of $19.6 \%$ favoring line in context was large and reliable $[t(7)=4.70, p<.01]$.

These data provide evidence that a substantial con- text effect can be observed even if the context is composed of no more than a 90-deg angle. The results from the remainder of the study, with the fixation point location shown in Figure $4 a$, indicate that such an effect depends on the location of the fixation point. With this fixation location, subjects correctly detected the line alone on $77.0 \%$ of the presentations and in context on $74.0 \%$. Thus, the previously noted context-superiority effect is eliminated. The small advantage of the line alone is not reliable $[t(7)=1.23, p>.05]$.

\section{EXPERIMENT 5}

Experiments 2 and 4 demonstrate that the effectiveness of a context in facilitating detection of target line elements depends upon the point of fixation. In both studies, facilitation by context occurred only if fixation was removed from the point where target line elements and context line components converged. However, the movement of the fixation point in these experiments away from that intersection also placed it in an area free of any line detail (see Figures $2 b$ and $4 \mathrm{~b}$ ). Experiment 5 asked whether facilitation requires moving the fixation point away from any line detail or only away from the intersection of target and context lines.

In Experiment 5, the fixation point was moved away from the junction of the target and context line to the position used in the preceding studies, but the context was modified so that the fixation

\section{SET A SET B}

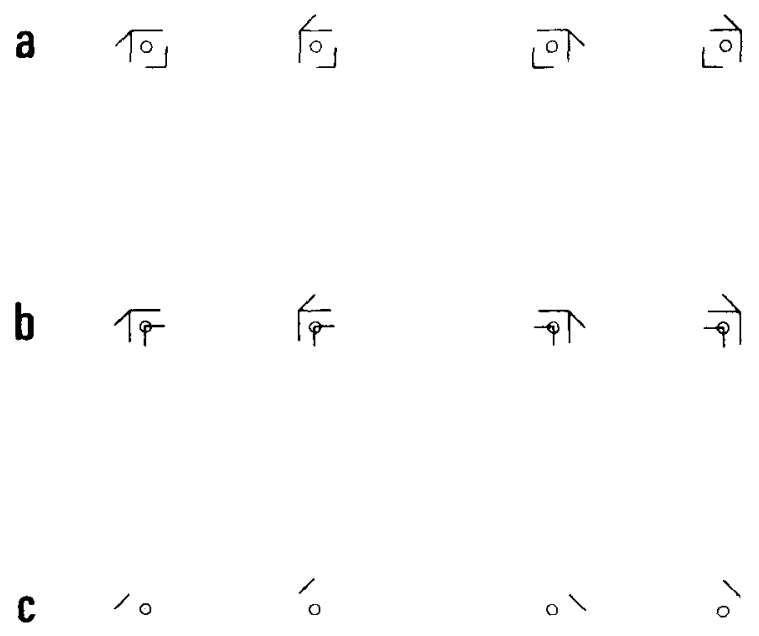

Figure 5. Target lines from Sets $A$ and $B$ are shown as part of Context 1 (a), where irrelevant line detail is removed from the point of fixation, as part of Context 2 (b), where irrelevant line detail falls upon the point of fixation, and as they appear alone (c). Circles are included only to clarify fixation point location; they were not part of stimulus display. 
point would fall upon either an area free of visual detail or one containing irrelevant line detail as shown in Figure 5. The corner context used in Experiment 4 is augmented by the addition of an irrelevant corner unit. In Context 1 (Figure 5a), the additional unit is positioned away from the fixation point. In Context 2 (Figure $5 \mathrm{~b}$ ), it is positioned to intersect directly with the fixation point. In neither case does it provide information about which target line is presented. If, however, the assumption is correct that it is the line detail at the fixation point, and not the movement of the fixation point per se, that is important, then target lines should be more difficult to detect in Context 2 than when presented alone, but easier to detect in Context 1.

\section{Method}

Stimuli. Context line segments and target line elements were the same lengths as in Experiments 2 and 4, and the same pen arrangement was used to draw the forms. The irrelevant line detail consisted of a 5-mm corner unit so positioned as to either intersect with or fall clear of the fixation point, as shown in Figure 5. The fixation point was located the same distance from the junction of context and target lines as in Experiments $2 \mathrm{~B}$ and 5 . Context forms and target line components subtended visual angles of .86 and $.56 \mathrm{deg}$, respectively.

Subjects and Procedure. An entirely within-subject design was used in an effort to provide a more sensitive assessment of impairment and facilitation effects, so all subjects received stimulus forms from both Sets A and B. Each of the three different types of forms (i.e., line alone and two different contexts) were presented in separate blocks (a change from preceding studies, in which all stimulus forms occurred in each block).

One-half of the subjects received all presentations from Set A first, and the other half received all presentations from Set B first. A complete presentation of visual forms in either set involved nine blocks of 32 presentations. Stimulus presentations within each block were random, and blocks representing the three different types of stimulus forms were randomly ordered, with the restriction that no one type of form (line alone or context) could be repeated until all three had been presented equally often. The experiment was carried out over two separate sessions. Each session was preceded by three blocks of practice presentations. Any adjustment in illumination level of the preexposure field to maintain the $70 \%-80 \%$ correct response level was made only after all three different types of stimulus forms had been presented equally often. The mean level of illumination of the preexposure field was $7.20 \mathrm{~cd} / \mathrm{m}^{2}$. The level of illumination and duration of exposure field were as in all preceding studies. Four naive subjects who had not served in any of the preceding experiments were recruited and paid for their services.

\section{Results}

Table 1 shows the proportion of correct detections of target lines alone or in Contexts 1 and 2 . The subjects were correct on $85.6 \%$ of the presentations of Context 1 , on $74.6 \%$ of the line alone, and on $67.2 \%$ of Context 2. The same ordering is evident in the data of every subject, both for Set A and Set B. Separate $t$ tests carried out on the number of correct responses confirm what is obvious from the table: Context 1 produced reliably better detection than did the line alone $[\mathrm{t}(3)=8.82, \mathrm{p}<.01]$, and detection in Context 2 was reliably inferior to detection with presentation of the line alone $[t(3)=5.36, p<.02]$. Differences cannot
Table 1

Proportion Correct Target Lines Detected in Sets A and B in Three Presentation Conditions

\begin{tabular}{ccccc}
\hline $\begin{array}{c}\text { Sub- } \\
\text { ject }\end{array}$ & $\begin{array}{c}\text { Target } \\
\text { Set }\end{array}$ & $\begin{array}{c}\text { Con- } \\
\text { text } 1\end{array}$ & $\begin{array}{c}\text { Con- } \\
\text { text } 2\end{array}$ & $\begin{array}{c}\text { Line } \\
\text { Alone }\end{array}$ \\
\hline 1 & $\mathrm{~A}$ & .823 & .656 & .740 \\
& $\mathrm{~B}$ & .792 & .588 & .729 \\
2 & $\mathrm{~A}$ & .854 & .677 & .771 \\
& $\mathrm{~B}$ & .875 & .719 & .729 \\
3 & $\mathrm{~A}$ & .875 & .677 & .740 \\
& $\mathrm{~B}$ & .875 & .688 & .760 \\
4 & $\mathrm{~A}$ & .927 & .708 & .740 \\
& $\mathrm{~B}$ & .823 & .667 & .760 \\
Mean & & .856 & .672 & .746 \\
\hline
\end{tabular}

be accounted for in terms of the greater apparent depth or coherence of Context 1 over Context 2. Reference to the Appendix shows that the less effective Context 2 was rated higher than Context 1 with respect to both depth $[\mathrm{t}(44)=4.21, \mathrm{p}<.01]$ and coherence $[\mathrm{t}(44)=6.04, \mathrm{p}<.01]$.

As in Experiments $2 B$ and 4, subjects were more accurate in detecting target lines when the fixation point was not located at the intersection of context and target lines. However, when irrelevant line detail was added to this location, context effectiveness vanished and, in fact, target lines were detected more accurately alone than in context. It is not the movement of the fixation point away from the convergence of target and context lines that increases context effectiveness, but rather the fact that the movement typically places the fixation point at a location that is free of line detail.

\section{GENERAL DISCUSSION}

\section{Depth, Coherence, and Context Effectiveness}

The initial objective of the study was to determine whether the effectiveness of a form as a context depends on the degree to which it represents a coherent and three-dimensional whole. Experiment 1 indicates that a form need be neither three-dimensional nor coherent to be an effective context. Both the dial and coordinate contexts used were judged to be lacking in depth, and the coordinate context was rated extremely low in coherence. Nevertheless, they were equally effective in facilitating target-line detection. The overall advantage of $10 \%-11.7 \%$ for lines in context over lines alone observed with these forms compares favorably with the $11.3 \%$ advantage for three-dimensional and coherent object forms reported by Williams and Weisstein in their Experiment 1 . The $16.6 \%$ advantage obtained with a slightly modified coordinate context exceeds the largest advantage reported by Williams and Weisstein. Additional doubt is cast upon the overriding importance of depth and coherence by the results of Experiments 2,3 , and 4 . The substantial changes in context effec- 
tiveness produced by moving the fixation point location in Experiment 2 could not be related to any corresponding change in apparent depth, and, in Experiments 3 and 5, the forms that were least effective were rated reliably higher in coherence and depth than the more effective forms. Clearly, threedimensionality and coherence are not properties essential for a demonstration that lines within forms can be discriminated more accurately than lines alone.

These findings are consistent with the recent report by McClelland and Miller (1979) that threedimensionality is neither a necessary nor a sufficient condition for the demonstration of context effects, and with the finding of Schendel and Shaw (1976) that constituent line units of individual letters (which are obviously two-dimensional) can be detected more accurately within the context of the letter than when presented independently. The findings are less compatible with the approach taken by Williams and Weisstein (1978) and Womersley (1977), which emphasizes the importance of three-dimensionality and coherence. Both may, under some circumstances, influence the magnitude of the context effect. Indeed, there is evidence that three-dimensionality can facilitate target line detection (Womersley, 1977; Weisstein, Williams, \& Harris, Note 1). However, the present studies clearly demonstrate that the context effect can be produced by a wide variety of contexts possessing little depth or coherence, and this fact must be dealt with in accounting for the effect or in utilizing it as an analytic tool.

\section{Context Effectiveness and the Fixation-Location Effect}

It is by no means clear why provision of a context should facilitate target line detection. As Weisstein and Maguire (1980) note, it is normally the case that the presence of adjacent lines impairs detection of target lines through simultaneous masking. One conceivable explanation is that the context serves as a reference frame to facilitate spatial location of target lines. It is certainly true that subjects cannot respond correctly unless they can effectively anchor the target line with respect to the fixation point. If, however, locating the target line with reference to the fixation point were the crucial problem, then the further the fixation point from the target line, the more difficult localization would be and the greater the benefit associated with the provision of a context. The data from Experiment 2, which involved such a manipulation, do not support this interpretation. The most pronounced context effects were not evident when the fixation point was farthest removed from target lines. Other evidence against such an interpretation of the context effect has been provided by Williams and Weisstein (1978), who reasoned that if the context assisted in pinning down the location of the target line with respect to the fixation point, then using two target-line elements falling along different diagonals (i.e., at a 90-deg angle to one another) should markedly diminish difficulties that might be encountered when target lines fall along the same diagonal, and that the context effect would thus be diminished or eliminated. This was not observed: The context effect remained pronounced. Another explanation must be sought, and, if it is to be adequate, it must explain not only how context facilitates targetline discrimination, but also why the facilitating effect of context varies as a function of fixation-point location.

Unexpectedly, fixation-point location turned out to be an important determinant of whether a context facilitated or impaired target line detection. In Experiment 2, for example, context facilitation was evident with the Williams and Weisstein threedimensional form only when the fixation point was located at the center of the forward square of the context. The object context impaired target-line discrimination when the fixation was located at the point of convergence of context and target lines. Similar effects were observed in Experiment 4. We sought at first to explain the fixation-location effect in terms of Sakitt's (1975) contention that the most enduring component of the visual image or icon produced in tachistoscopic experiments is rod-generated. Thus, a shift of the target and context lines to a less central location in the visual field could serve to increase the rod contribution to the icon and facilitate detection of target lines. However, there are several troubling aspects to this argument. For example, it is difficult to believe that any increase in the duration of the icon would do more than compensate for the acuity loss occasioned by a substantial peripheral shift. Indeed, in Experiment 2, in which there was such a shift of the fixation point, there was no advantage associated with the provision of a context. Furthermore, Experiment 5 suggests that it is not only the movement of the fixation point that is important, but also the character of the line detail on which the fixation point rests. If context and fixation location effects are to be explained, it is necessary to go beyond the assumption that a context serves simply as a reference frame to facilitate spatial location and that the fixation location effect can be explained in terms of the involvement of the scotopic system.

\section{Potential Role of Global and \\ Local Processing Systems}

In trying to account for context and fixation location effects, our inclination is to follow the lead of Broadbent (1977) and Navon (1977), who argue that both global and local analytic systems are involved in the perceptual analysis of form. The global system is assumed to provide what amounts to a low-resolution rendering of the overall visual terrain. It precedes, and is assumed to provide a foundation for, the more detailed local analysis which follows. ${ }^{2}$ The line-incontext effect can be explained in the same manner as 
the word superiority effect (Navon, 1977). That is, the prior availability of the global configuration provides information that can facilitate local analytic operations involved in attending to, discriminating, and encoding the target line.

While the global system is assumed to be critical to the context effect, the fixation-location effect is most likely attributable to the way in which the local analytic system operates. Our findings in Experiments 2, 3, and 4, that contexts are most effective when the fixation point is removed from complex line intersections, and in Experiment 5, that adding irrelevant line detail to the point of fixation impairs context effectiveness, lead us to suggest that (1) there are preferred ways of executing the local analytic sequence, that is, in analyzing and understanding the structure of the context presented, and (2) that in tachistoscopic presentations the analytic process starts at the point of fixation. This means that the location of the fixation point in a context is extremely important. Unless the experimenter has placed the fixation point so that it falls upon the location that would represent the first step in the preferred analytic sequence, there will be interference and impairment of the course of analysis of the context. Since it is unlikely that the experimenter will be able to anticipate the preferred processing sequence, impairment is more likely than facilitation. If, however, the fixation point falls on a clear area or a point with a minimum amount of line detail, there should be far less difficulty in digesting the detail present and pursuing a course of analysis that conforms more closely to the preferred sequence.

The view that a preferred analytic processing sequence starting at the point of fixation is involved in the perception of simple outline forms may appear implausible, but it is difficult to explain, without such an assumption, why a given context should impair detection of the target line when the eyes are fixated at the junction of target and context lines and facilitate target line detection when the eyes are fixated at a detail-free area a short distance away. It is target line detection that is the objective, in the experiments reported, and common sense suggests that having the target line at the point of fixation should produce the most effective performance. The fact that it does not, but, rather, leads to impaired target line detection, persuades us that subjects may have difficulty in carrying out analytic processing sequences necessary for comprehension of a form when that form is briefly glimpsed from certain fixation point locations. This view is strengthened by the finding that the object-superiority effect can be shown also to depend crucially on the location of the fixation point (Earhard \& Armitage, Note 2). Other data on the word-superiority effect provided by Johnston and McClelland (1974), indicating that having a subject look at the specific location in a word where the crucial target letter will appear leads to less effective performance than allowing subjects the use of an unconstrained viewing strategy, suggest also that there may be a preferred processing sequence which can be impaired by altering fixation point location.

This analysis is an admittedly after-the-fact effort to attempt to account for context and fixation-location effects. It is speculative and it has deficiencies. There is, for example, no way of specifying at this time what the preferred order of processing will be for a given form, or class of forms, and hence no way of knowing what the effect of a particular fixation point location will be. In fact, it is conceivable that, for some forms, the local analytic process may begin most effectively at a point where line detail is located. This could account for a report by McClelland and Miller (1979), in which context facilitation is observed with the fixation point resting on line detail. What is required to clarify these issues is a systematic analysis of the interaction between fixation point location and the amount and type of visual detail on, or near, the fixation point for a wide variety of forms. We are currently attempting to provide such an analysis.

\section{REFERENCE NOTES}

1. Weisstein, N., Williams, M. C., \& Harris, C. S. Line segments are harder to see in flatter patterns: The role of threedimensionality in "object-line" and "object-superiority" effects. Paper presented at the meeting of the Psychonomic Society, Phoenix, November 1978

2. Earhard, B., \& Armitage, R. Context effectiveness in perception: Is object superiority a consequence of the fixation location effect.' Manuscript submitted for publication, 1980.

\section{REFERENCES}

Broadbrint, D. E. The hidden preattentive process. American Psuchologist, 1977, 32, 109-118.

Johnston, J. C., \& McCielland, J. L. Perception of letters in words: Seek not and ye shall find. Science, 1974, 184, 1192-1194.

MCClfilland, J. L. Perception and masking of wholes and parts. Journat of Experimental Psychology: Human Perception and Performance, 1978, 4, 210-223.

MCClfiliand, J. L., \& Millek, J. Structural factors in figure perception. Perception \& Psychophysics, 1979, 26, 221-229.

Navon, D. Forest before trees: The precedence of global features in visual perception. Cognitive Psychology, 1977, 9, 353-383.

Saki'T, B. Locus of short-term visual storage. Science, 1975 , $190,1318-1319$

Schindit, J. D., \& Shaw, P. A test of the generality of the word-context effect. Perception \& Psychophysics, 1976, 19 , $383-393$

Weisstrin, N., \& Harris, C. S. Visual detection of line segments: An object-superiority effect. Science, 1974, 186, 752-755.

Weisstein, N.. \& Maguire, W. Computing the next step: Psychophysical measures of representation and interpretation, In E. M. Riseman \& A. R. Hanson (Eds.), Computer vision svstems. New York: Academic Press, 1980.

Williams, A., \& Wrisstrin, N. Line segments are perceived better in a coherent context than alone: An object-line effect in visual perception. Memory \& Cognition, 1978, 6, 85-90.

WomersLey, M. A contextual effect in feature detection with application of signal detection methodology. Perception \& Psy. (hophwsis, 1977, 21, 88-92 


\section{NOTES}

1. Through an oversight, separate depth ratings were not obtained for the two different fixation-point locations used. Ratings were obtained subsequently from 20 naive subjects using the same procedures described in the Appendix. The mean depth ratings of 6.10 with fixation at the intersection of target and context lines and of 6.35 with the fixation point moved the appropriate distance away were slightly higher than those obtained with no fixationpoint specification, but they did not differ reliably $[\mathrm{t}(19)=1.20$, $p>.051$. There is no basis, therefore, for attributing observed differences to a change in apparent depth produced by a shift in fixation-point location.

2. Broadbent (1977) and Navon (1977) relate the global and local analytic systems to the activity of low and high spatial frequency channels and cite evidence to support the view that the global (low-frequency) channel is more rapidly activated than the local (high-frequency) channel.

\section{APPENDIX}

\section{Depth and Coherence Ratings of Contexts Used}

All forms used in the experiments reported were rated for coherence and three-dimensionality. Additional ratings were obtained for the Williams and Weisstein object form, with the fixation point located at the three different positions used in Experiment 2, to ascertain whether apparent depth varied with fixation-point location.

\section{Method}

Subjects. A total of 90 undergraduates were used as subjects. All received course credit points for participating.

Stimuli. Fifty-seven stimuli were rated: 24 represented all four variants of the contexts used in the studies reported, and the remaining 33 were contexts used, or intended to be used, in other studies. With one exception, only ratings for forms from the present study will be reported. The exception is Williams and Weisstein's line context (their Figure 1d). It was included to provide a basis for comparing ratings of our forms, which were considered deficient in depth and coherence, with a commonly used form held to have similar deficiencies (see Weisstein \& Harris, 1974; Weisstein \& Maguire, 1980). The same booklets were used to obtain depth and coherence ratings. The booklet contained the 57 stimuli described above. Only a single exemplar of a given form was contained on each page, and opposite each form was a line for subjects to enter the assigned rating. A subsidiary booklet was constructed to determine whether there were any changes in apparent depth associated with the movement of the fixation point to different locations in Williams and Weisstein's object form in Experiment 2. Each page of the subsidiary booklet contained a single form. On the right of each form was a space to enter the assigned rating, and on the left was a single square with an $\mathrm{X}$ signifying the point on which subjects were to fix their gaze on the forward square of the three-dimensional form while making their judgments.

Procedure. Independent groups of subjects rated for coherence and for three-dimensionality. Booklets were administered to groups of $10-20$ subjects at a time. The subjects who rated coherence received a written description which informed them that coherence reflected the degree to which parts of a form combined to form an integrated and unitary whole and that forms high in coherence had a unity that made them distinctive and readily distinguishable from other forms, even under adverse viewing conditions. They were instructed to use this information as a guide in rating individual forms on a scale running from zero, which represented the lowest conceivable level of coherence, to 10 , which represented the highest possible degree of coherence.

Subjects rating for depth first received the large booklet containing 57 stimuli. They were presented with a 0 -to-10 scale, with a two-dimensional square at one end and a three-dimensional cube
Table 2

Mean Depth and Coherence Ratings (on a 0-10 Rating Scale) of 12 Contexts

\begin{tabular}{lcccr}
\hline & & \multicolumn{2}{c}{ Rating } \\
\cline { 3 - 5 } \multicolumn{1}{c}{ Context } & $\begin{array}{c}\text { Experi- } \\
\text { ment }\end{array}$ & Depth & $\begin{array}{r}\text { Coher- } \\
\text { ence }\end{array}$ \\
\hline Dial (Figure 1a) & 1A & 1.27 & 7.24 \\
Coordinate (Figure 1b) & $1 \mathrm{~B}$ & 2.68 & 3.64 \\
Object* & 2 & 8.42 & 7.99 \\
Object (Fixation as in Figure 2a) & 2 & 7.29 & \\
Object (Fixation as in Figure 2b) & 2 & 6.70 & \\
Object (Fixation as in Figure 2c) & 2 & 6.75 & \\
Line* & & 2.53 & 4.25 \\
Large Coordinate (Figure 3a) & 3 & 2.68 & 3.64 \\
Small Coordinate (Figure 3b) & 3 & 3.33 & 4.22 \\
Corner (Figure 4) & 4 & 5.55 & 4.34 \\
Corner Context 1 (Figure 5a) & 5 & 5.79 & 4.14 \\
Corner Context 2 (Figure 5b) & 5 & 6.88 & 5.74 \\
\hline
\end{tabular}

"The "Object" and "Line" forms from Williams and Weisstein (1978) were used as baseline contexts to evaluate threedimensionality and coherence ratings of other forms used in the experiments reported (see text).

at the other, and were told to use this scale as a guide in rating variations in depth among the forms. After completing the large booklet, they were handed the second subsidiary booklet and informed that we were interested in knowing whether there was any change in the apparent depth or three-dimensionality of forms when they were examined with the eyes positioned at different locations. Explanations were given about how to fix their eyes when making judgments. Subjects were questioned to make sure they understood the instructions and were reminded during the course of the task to make certain their eyes were fixed on the point specified. Subjects making both kinds of ratings were informed that they would encounter different variants of the same form and that they were to consider each one independently in assigning a rating.

\section{Results}

The ratings assigned to all variants of a given form were averaged over subjects and are shown in Table 2. Detailed results of statistical comparisons are provided in the description of individual experiments, where the outcome of such comparisons is relevant to the interpretation to be placed on the data obtained. Only the general point will be made here that, on the whole, the pattern of results conformed with our expectancies. The Weisstein and Williams object form received the highest depth and coherence ratings, as should be the case, given that it is viewed as a prototypical example of a coherent and threedimensional context. Similarly, the line context of Weisstein and Williams, which is widely utilized as an example of a form lacking depth and coherence, was rated low on both dimensions. Differences between the object and line contexts in both depth and coherence are large and reliable $[\mathrm{t}(44)=14.01, \mathrm{p}<.001$, and $\mathrm{t}(44)=7.85, \mathrm{p}<.001$, respectively]. Thus, our rating procedures were sufficiently sensitive to detect differences conventionally assumed to exist between the object and line contexts, and the depth and coherence values assigned these contexts can serve as a basis for relating the values assigned to our forms to those assigned to forms used conventionally in context studies.

(Received for publication June 15, 1979; revision accepted April 4, 1980.) 